\title{
An Exemplar Based Method for Inpainting of an Image
}

\author{
Nikku Ravi ${ }^{1}$, Kapil Kumar Nagwanshi ${ }^{2}$ \\ ${ }^{1}$ M. Tech Scholar, Department of Computer Science and Engg, Rungta College of Engineering and Technology, Bhilai, Durg (CG) India \\ ${ }^{2}$ Associate Professor, Department of Computer Science and Engg, Rungta College of Engineering and Technology, Bhilai, Durg (CG) India
}

\begin{abstract}
The research paper presented here involves Image inpainting techniques and texture synthesis method for the hole filling in an images. It also involves attaining of the similar texture as of the background after removal of the part of an image. It can be also considered as the image restoration method, since it can be implemented for image restoration of a degraded image. Texture synthesis is the process by which large image regions can be generated from the best sample texture. Inpainting techniques involve filling of small image gap. Image restoration techniques are generally based on mathematical or probabilistic method. When both inpainting process and texture synthesis process are adopted for image enhancement the enhanced image obtained is much original and flawless. The process required for the replication of both texture and structure is well represented by exemplar based inpainting.
\end{abstract}

Keywords: Image Restoration, Image Inpainting, Texture Synthesis, Patch Priority, Region filling.

\section{Introduction}

According to the definition [1] use of digital computers for the processing of images refers to a process known as digital image processing. A detailed study of digital image gives us an overview of its composition. It consists of elements each of which has a particular location and value. This element can be referred or are generally referred by as picture elements, image elements, pels and pixels. Images are obtained from various sources. These digital images are generally used by different people for various purposes. According to the varying needs, images can be altered by the user. Some part of images may get detroited. Today in this digital world all digital images are stored digitally which can be altered in different ways. Also the old images may develop cracks. Others may have text embedded on it. This undesired part of the image can be removed by creating large holes.

Object removal on digital photos that is removal of occlusions (date, stamps, logo etc), large unwanted regions, red eye corrections, super resolutions, restoration of old films and paintings etc can be done by the application of a technique known as Inpainting [2].

Image inpainting has come up with many applications in the digital world. It is being used for creating special effects by removing unwanted objects from the image. These unwanted objects can range from microphone, ropes, undesired persons and logos, stamps, dates, and texts in the image. There may be part of images which could be missing during the transmission process over the network. These can be reconstructed by using image inpainting. Decomposition of original image is done on the structure and texture image. Each image's reconstruction is performed separately.

Partial Differential Equation(PDE) based algorithm are mostly designed to connect the edges or to extend level lines in some adequate manners into inpaiting domain[3][4].This kind of algorithm is targeted at extrapolating geometric image features, especially edges. But this algorithm produce many disturbing artifacts if the inpainting domain surrounded by texture regions. A sample of the available image data is being used by the texture synthesis algorithm which aims at filling the inpainting domain such that the color relationship statistics between neighbourhood pixel matches those of the sample[3][4].It aims for creating intra region details. Here if the inpainting domain is surrounded by differently textured regions then it may produce artifacts. Many research work has considered texture synthesis as a way to fill large regions with pure textures and repetitive two dimensional textural patterns with moderate stochaticity[5]. Exemplar based techniques can cheaply and effectively generate new texture by sampling and copying colour values from the source [6].

But these techniques are not as easier in filling holes in photographs of real world scene which often consists of linear structures and composite textures, as effective they are in replicating consistent texture [7]. Boundaries between the image regions are complex product of manual influences between different textures are the chief difficulties here. Compared to two dimensional nature of pure textures, these boundaries form what might be considered more onedimensional, or linear, image structures.

Algorithms specifically addressing all these issues for the task of image restoration, where speckles, scratches and over laid text are removed by propagation of linear structures i.e isophotes in inpainting literature in to the target region via diffusion[8][9].

One of the algorithm based on the priority distribution based on colour distribution[10] is also established in the field of exemplar based image inpainting.

Mostly these are inspired by the the partial differential equations of physical heat flow, and work convincingly as restoration algorithms. They come with a drawback that the diffusion process introduces Some blur, which is noticeable when the algorithm is applied to fill larger regions. 


\section{International Journal of Science and Research (IJSR) \\ ISSN (Online): 2319-7064}

Index Copernicus Value (2013): 6.14 | Impact Factor (2015): 6.391

In the algorithm presented combines the strength of both the approaches. In inpainting special attention is paid to linear structures. The linear structure adjoining target region only influence the fill order of what is at core an exemplar based texture synthesis algorithm. It results in an algorithm that has efficiency and qualitative performance of exemplar based texture synthesis and also respects the image constraints imposed by surrounding linear structures.

\section{Methodology}

\subsection{Exemplar based synthesis suffice}

The work done here adopts features of [1] which decomposes the original image into two components: inpainting is done on one component while the other component is done with texture synthesis. Thus the summation obtained by the two processed image is the output image, obtained as the result. Isophotes driven image's sampling is the chief part of the algorithm majorly being used [11]. Exemplar based images perform well on two dimensional textures. The interaction of the interface of two texture is done by a special solution for this purpose which is known as Algorithm for parallel synthesis of composite texture [12]. The synthesis of both pure and composite texture is generated by a single mechanism, which also considered sufficient for it.

The target region denoted by $\Omega$ is to be filled and the contour of this target region is denoted by $\delta \Omega$. With the progression of the algorithm an inward evolution of the contour is generated which is known as fill front. $\Phi$, the assumed source region remains fixed throughout the algorithm and it provides samples used for the filling process. A single iteration process is being processed, which is the focus of the algorithm. This shows how the texture and structure are handled adequately by exemplar based synthesis. If a square template $\Psi \varepsilon \Omega$ center at any point is to be filled. The most apt match for the sample is provided by the patch $\Psi$ ' $\varepsilon \Phi$. Then the source region which seems to be the most analogues to those regions is filled in $\Psi$.

It is mostly considered as a simple transferring of the patterns from the best match source patch that is the inward propagation of the isophote. Preservation of the orientation of isophote is thus conserved automatically.

\subsection{Algorithm for region filling}

The foremost task is that of the user, for the selection of the target region, $\Omega$, which is to be removed and filled with new information. We can present $\Phi$ the source region as the entire image minus the target region $(\Phi=I-\Omega)$. This can be represented as a dilated band around the target region, or it may be manually specified by the user.

As usually with all texture synthesis [13], the defined dimension of the template window $\Psi$ must be precise. A window size of $9 \times 9$ pixels which is usually considered as default is to be provided. In general practice it is required by the user to lay down this size a bit larger than the one with the largest distinguishable texture element, or texel of the source region. As all these parameters are once resoluted then the remainder of the region filling process becomes automatic on the whole.

The algorithm being adopted maintains a colour value and a confidence value for each pixel. These fixed values reflects the pixel value. Once these has been filled then it is given a frozen state. A temporary priority value is assigned to the patches which are along the fill front. This value determines the order which has been utilized for filling it. Further the algorithm moves with the following procedure till all the pixels get filled.

\subsection{Patch priorities computation}

Non parametric texture synthesis' crucial part is filling order[14]. The synthesis of the target region from the outside to inward pattern in concentric layers, being the most default method used and is known as onion peel method 15]. It is considered as one of the general method.

Another patch based filling algorithm is based on the concept of sparsity[16] demonstrating the concept of sparsity at patch level.

The algorithm performs the task of filling of the patches through the best first filling algorithm. This process is entirely dependent on the priority values. These are the valued which is being given to each patch on the fill front. The computation of the priority is generally deflected towards the patches which are in the extension of the strong edges and are also surrounded by pixels with high confidence.

Let a patch $\Psi$ centered at the point $\mathbf{p} \varepsilon \delta \Omega$ whose priority $\mathrm{P}(\mathbf{p})$ is defined as the product of two terms in equation 1 :

$$
\mathrm{P}(\mathbf{p})=\mathrm{C}(\mathbf{p}) \mathrm{D}(\mathbf{p})
$$

where $C(\mathbf{p})$ is the confidence term and $\mathrm{D}(\mathbf{p})$ the data term, and they are defined as in equation 2 :

$$
C(\boldsymbol{p})=\frac{\sum_{q \in \Psi_{\cap} \bar{\Omega}} C(\boldsymbol{q})}{\Psi}, D(\boldsymbol{p})=\frac{\left|\nabla I_{p} n_{p}\right|}{\alpha}
$$

here $|\Psi|$ is the area of $\Psi, \propto$ is defined as normalization factor and $n_{y}$ is the unit vector which is orthogonal to the front $\delta \Omega$ in the point p. Every border patch is calculated with discrete patches for individual pixel that are present on the boundary target region The function $\mathrm{C}(\mathbf{p})$ is set to zero $\forall_{p} \in \Omega$, and $\mathrm{C}(\mathbf{p})=1 \forall_{p} \in \mathrm{I}-\Omega$ at initial step.

The foremost task performed in this step is to load up those regions whose most part of the pixel is filled and also to those pixels which were filled earlier.

Thus $C(p)$ roughly introduces the desirable concentric fill order. Pixels those present in the outer layer comes up with comparatively greater confidence values and thus are filled earlier.

Data term $D(p)$ is regarded as the function of strength of isophotes which are hitting the front patch at each iteration. 


\section{International Journal of Science and Research (IJSR) \\ ISSN (Online): 2319-7064}

Index Copernicus Value (2013): 6.14 | Impact Factor (2015): 6.391

This term has the function of boosting up the importance of a patch than an isotope flows into.[17]. A balance which is considered delicate and fragile is maintained between the data term and confidence term significantly. Data term which significantly tries up to press on the isophote inwards, while the confidence term tend to restrain accurately this sort of invasion into the target region. This fragile balance is handled by the mechanism that is the single priority computation for all patches on the fill front. The fill order being used up here is the action of image properties resulting in an organic synthesis process that is able to eliminate the risk of broken structure, artecrafts and also suppresses those blocky artecrafts without an expensive patch cutting step [18] or a blur inducing blending step [19].

\subsection{Structure information and texture propagation}

After the computation and calculations of all the priorities of the fill front is done then the patch which is having the highest priority is obtained. This computed patch is then done with the data that is extracted from the source region. Traditional image inpainting uses image information propagation through diffusion leading to smoothing of image. This results in blurry fillings especially for larger regions.

Thus the direct sampling process is adopted for the source region for the propagation of the image texture. Search is proceeded in similar manner as in [13] for the patch that is extremely similar to the patch priority $\left(\Psi_{\mathrm{p}}\right)$. Thus we have equation 3 as:

$$
\Psi_{q}=\arg \underbrace{\min }_{\Psi_{q} \in \Phi} d\left(\Psi_{p}, \Psi_{q}\right)
$$

where $\mathrm{d}\left(\Psi_{a,} \Psi_{b}\right)$ is the distance between two general terms. $\Psi_{a}$ and $\Psi_{b}$ are the representation of the sum of squared differences (SSD) of those pixels which have been already filled in the two patches described earlier. The calculated value of each pixel which is to be filled, $p^{x} \mid p^{y} \in \Psi_{p n n}$, is derived from its corresponding position which is inside $\Psi_{\mathrm{qq}}$. The source exemplar $\Psi_{q}$ is utilized for this filling purpose.

After the $\Psi_{\mathrm{p}}$ patch has been filled with new pixel value then the upadation of the confidence $C(p)$ in the area is delimited as in the equation 4 :

$$
\mathrm{C}(\mathbf{q})=\mathrm{C}(\mathbf{p}) \forall q \in \Psi_{\mathrm{p}} \cap \Omega \text {. }
$$

The rule for the updation which has been discussed in the earlier section helps for the measurement of the relative confidence for the patches created on the fill front. This is done in the absence of parameters specific to the images. With the advancement of the filling process, the confidence value starts to get decay. This decay indicates a reduced assurance of the values of color for the pixels that are closer to the centre of the target region.

\section{Proposed Algorithm}

The algorithm proposed has several steps, which consist of combination of several image processing method.

The algorithm consists of following steps and functions:
1. Confidence value and the colour value, a general basic property, maintained by each pixel or an empty or null value for those pixel which is unfilled.

2. The calculation and computation of the patch priorities as in accordance with the equation 5 is the first step. It includes computing the patch priority $P(p)$. These are biased towards those which are in continuation with the strong edges and are surrounded by strong edges.

3. $P(p)=C(p) D(p)(5)$

4. The next step involves the propagation of the texture and imparting of the structure information.

5. The selection of the patch is based on the factor is the one with highest priority is chosen for the propagation.

6. The basic factor on which the priority of any given pixel are based on the confidence term and data term.

7. At the end updation of the confidence value is done. Below flowchart shows the proposed algorithm:

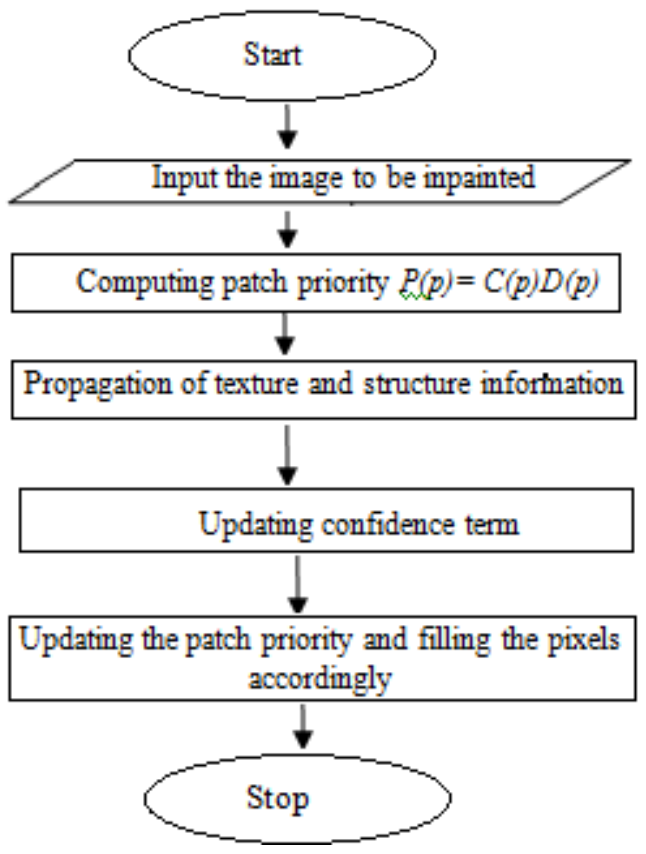

\section{Results and Discussion}

The algorithm being discussed has been applied to a variety of images ranging from purely synthetic images to full colour photographs that have been including complex texture. The general crieteria being followed all over the algorithm is the setting of the patch size to be greater than the largest texel present or the structure which has been the thickestin the source region.

Mostly all the examples presented has source region set as $\Phi$ $=I-\Omega$.

The real advantage of this approach can be apparentely best seen in images which are real photographs with large ojects to remove. Fig(1) shows the example of a bungee jumper. Here the jumping man is the object to be removed. The shore line and the edge of the house, like structures have been automatically propagated into the target region along the plausible textures of shrubbery, water and roof tiles. All this is done with no priori model of anything specific to this image. 


\section{International Journal of Science and Research (IJSR) \\ ISSN (Online): 2319-7064 \\ Index Copernicus Value (2013): 6.14 | Impact Factor (2015): 6.391}

The topological changes of the target region is handled effortlessly while filling process. it can be observed that the filled region prominenetly mimics the surrounding region with not much prominent artefacts.

The PSNR for this image is 62.3292 and MSE is 0.0383 . The algoriithm being appplied is lack of diffusion step resulting in a blur free image. thus making the algorithm more computatonaly efficient.
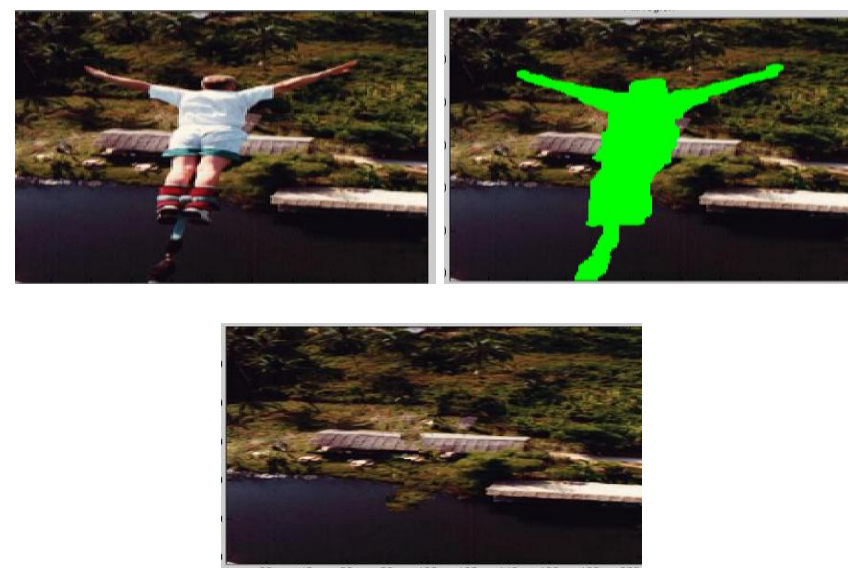

Figure1: bungee0.png

The next example which has been demonstrated in Fig(2) shows the application of the algorithm for the the images with different texture. In the photographic image presented here is a man skieng. The image is having variant texture of snow, building. wood etc. The target region is selected, that is the removal of the man and poles to get an image wihout a man. The object to be removed is first coloured then the algorithm is applied.

The PSNR obtained is 62.1783 and MSE is 0.0397 for the image of man on snow.
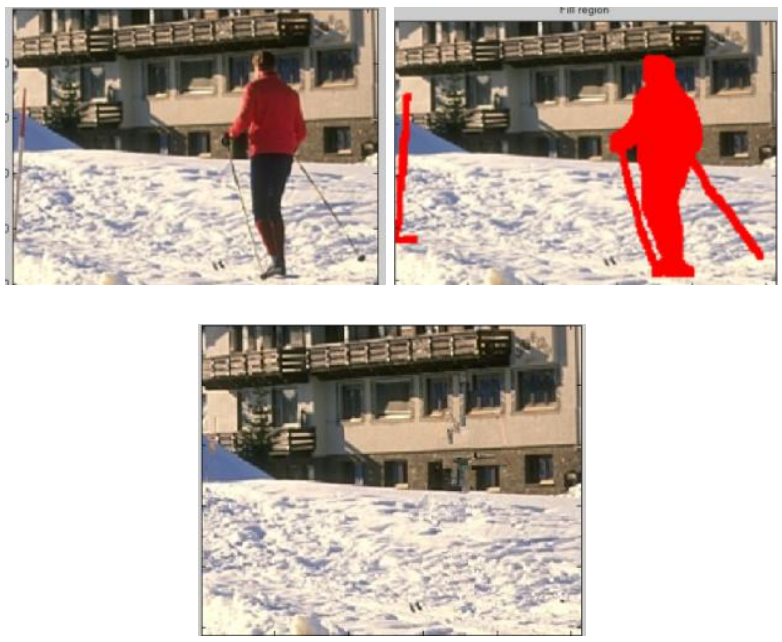

Figure 2: man.png

Another example can be seen of a wolf in the snow and wood in he background. the PSNR of the recovered image is 68.6627 and MSE is 0.0089.
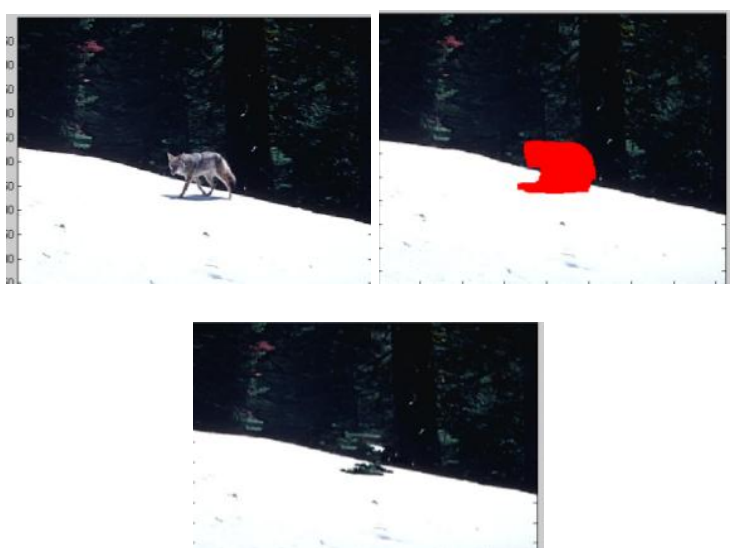

Figure 3: wolf.png

Table 1: PSNR

\begin{tabular}{|c|c|c|}
\hline Image name & Present & Earlier method \\
\hline bungee & 62.3292 & 35.42 \\
\hline man & 62.1783 & 34.77 \\
\hline wolf & 68.6627 & 36.53 \\
\hline
\end{tabular}

\section{Conclusion}

The purpose of this paper is the removal of the large objects from the digital image and photographs. A modification based exemplar image inpainting algorithm is adopted for this purpose. By this algorithm for the purpose of removal of object the result is an image in which the selected object is replaced by the undistinguishable background image that would be mimicking the source region.

This algorithm would be enhancing the robustness and effectiveness by including image gradient information. The filling order of the region along with the exemplar based texture synthesis technique is the scheme utilized for the determination of the filling order target. The natural images used as input for the algorithm demonstrates us that the developed algorithm can reproduce texture and as well as the structure of the surrounding area of the inpainted region. It has also been found useful in completion of an image, which has hole due to distortion of the image, by filling the hole and accurate propagation of textured pattern. The result obtained is much more preferable.

The technique introduced is capable for the propagation of both lineared structured and two dimensional texture into the target region.

Further work for object removal and image inpaiting from video images are the scope of this algorithm.

\section{References}

[1] Digital image processing by Rafael C. Gonzalez and Richards E. Woods.

[2] Bertalmio M, Vese L, Sapiro G ,Osher S. "Simultaneous structure and texture image inpaintings", IEEE transactions on Image processing,2003,12,882889.

[3] Chan T, Shen J, "Local inpainting models and TV inpainting ", SIAM journal on applied Mathematics ,2001,62, 1019-1043.

\section{Volume 5 Issue 6, June 2016}

www.ijsr.net 


\section{International Journal of Science and Research (IJSR) \\ ISSN (Online): 2319-7064}

Index Copernicus Value (2013): 6.14 | Impact Factor (2015): 6.391

[4] Chan T, Shen J, "Non texture inpainting by curvature driven diffusions", Journals of visual communication and Image representation, 2001,4, 436-449.

[5] L.W .Wey and M.Levoy, "Fast texture synthesis using tree structured vector quantization", In proceedings of ACM conf. Comp.Graphics(SIGGRAPH), 2000.

[6] A.Hertzmann, C.Jacobs, N.Oliver, B.Curless and D.Salesin, "Image analogies", In proceedings ACM Conf. Comp. Graphics(SIGGRAPH), EugeneFiume, Aug 2001.

[7] A.Talesny, V.Ferrari, G.Caenon and L.Van Gool, "Parallel composite texture synthesis", in texture 2002 workshop(in conjuction with ECCV02), Copenhagen, Denmark, June 2002.

[8] K.Sangeetha, Dr P.Segottuvelam and E.Balamurugan, "Improved exemplar based texture synthesis for natural scene completion", IJCSI International Journal of Computer Science Issues, Vol 8, Issue 5, No 2, September 2011.

[9] M.Bertalmio, A.L Bertozzi and G.Saprio,"Navier stokes, fluid dynamics and image and video inpainting" in proceeding Conf. Comp. Vision pattern Rec., pp.I:355-362, Hawai, Dec 2001.

[10] Qing Zhang and Jiajun Lin, "Exemplar based image inpainting using color distribution analysis", Journal of Information Science and Engineering 28, 641654(2012).

[11] M.Prasad, G.Mary Prakash Kumari and B V Raja Rao,'Image inpainting using texture synthesis", National Conference on Research Trends in Computer Science and Technology, 2012.

[12] A.Zalesny, V.Ferrari, G.Caenon and L.Van . Gool,"Composite Texture Synthesis", International Journal of Computer Vision Volume 162, Issue 1-2, pp 161-176, 2005.

[13]A. Efros and T. Leung, "Texture synthesis by non parametric sampling", in proceeding ICCV, pp 1033-138, Kerkyra, Greece, Sep 1999.

[14] Bowden, Richard , and Collomosse, John and Mikolajczyk, Krystian, "Non parametric synthesis of laminar volumetric texture.", proceedings of the British Machine Vision Conference 2012 pp 54.1-54.11

[15] www.google.com , last reference date 22 January 2015.

[16]Zongben Xu and Jian Sun,"Image inpainting by patch propagationusing patch sparsity.",IEEE transaction on image processing, VOL. 19, NO. 5, May 2010.

[17] T. F Chan and J.Shen, "Non texture inpainting by curvature driven diffusions", J.Visual Comm.Image rep.,4(12),2001.

[18] A.Efros and W.T Freeman,'Image quilting for texture synthesis and transfer" in proceeding ACM Conf. Comp.Graphics(SIGGRAPH).

[19] L.Liang, C.Liu, Y.Q.Xu, B.Guo and H.Y.Shum,"Real time texture synthesis by patch based sampling" in ACM Transactions on Graphics, 2001. 